\title{
Using Constrained Model Predictive Control to Control Two Quadrotors Transporting a Cable-Suspended Payload
}

\author{
Yaser Alothman \\ School of Computer Science \\ and Electronic Engineering \\ University of Essex, Colchester, UK \\ University of Technology, Baghdad, Iraq. \\ Email: ynialo@essex.ac.uk
}

\author{
Dongbing $\mathrm{Gu}$ \\ School of Computer Science \\ and Electronic Engineering \\ University of Essex, Colchester, UK \\ Email: dgu@essex.ac.uk
}

\begin{abstract}
This paper applies a constrained MPC controller to control two quadrotors which carry a cable-suspended payload together. The system dynamics is derived from the Euler-Lagrange equation. Given the dynamics complexity, a linear MPC controller is employed for a control task, which is to make the payload to track a desired trajectory while stabilising the two quadrotors. The constraints on quadrotor control signals and payload positions are taken into consideration. The constrained controller could be useful for practical control systems. The simulation results are provided to evaluate the control performance against an LQR controller.
\end{abstract}

\section{INTRODUCTION}

The research on Unmanned Aerial Vehicles (UAVs) transporting payloads by cables is growing worthily with a wide range of potential applications. In recent decades the maturation has been seen for civil and military applications in different research areas such as emergency rescue, reconnaissance, firefighting and freight transportation missions. The problem of multiple quadrotors transporting a cable-suspended payload is still challenging due to the complexity of systems.

Carrying a cable-suspended load by one, two, or multiple quadrotors have been investigated recently using various advanced control techniques such as linear and nonlinear controllers. A geometric controller was constructed to achieve the system stability of controlling a cable suspended payload with multiple quadrotors following a desired trajectory in [1], where a geometric feedback controller was designed initially for a reduced system then extended to the full system through singular perturbation method. Cooperative transportation of a cable suspended load was presented by the authors in [2] and [3] considering the tracking controller based on a coordinate-free model.

Those non-linear controllers are able to stabilise the control system but the dynamic performance cannot be optimised. Furthermore, the constraints on system states and input signals are not considered. In practical applications, it is necessary to handle these constraints for a control system in order to achieve a better control performance. The constraints to be considered in such a system could include the payload angles, positions, or quadrotor thrusts.

Model Predictive Control (MPC) has emerged as one of the most popular and valuable control techniques since the 1980s. The strategy of an MPC controller is to find a sequence of optimal controlling signals in a finite optimization horizon first and then select the first one to control the system recursively at each time step. It can not only optimise the cost function but also take the state or input constraints into consideration.

Many MPC studies are proposed for single quadrotors in two or three dimensions to implement different tasks and improve the system performance. The simplified models led to the design of a robust MPC controller in [4] to control the attitude of single unmanned quadrotor helicopter in severe environmental conditions based on piecewise affine linear systems. The challenging issue is to consider the wind-disturbance when executing sudden manoeuvres. The constraint on input signals was taken into account of this system. In [5] two control algorithms LQR and MPC were presented based on linear quadratic technique and implemented on a quadrotor testbed (Qball-X4) to control against the loss of control effectiveness with a faulttolerant control strategy. A linear MPC was proposed in [6] and the model was simplified with only two-dimensional movement based on the least square identification. An integral MPC controller was applied for the autonomous flight of single quadrotor to improve the translational motion tracking performance in [7]. The system was modelled based on a set of Piecewise Affine (PWA) systems and the physical constraint was considered. In manoeuvring over constrained environments, a robust MPC controller was presented in [8] for single quadrotor with a fixed payload to minimize the predicted tracking errors.

Cooperative UAV control in a form of multiple team formation was accomplished under a linear MPC control law [9] using the Qball-X4 quadrotor. This approach was implemented without constraints and no load considered. 


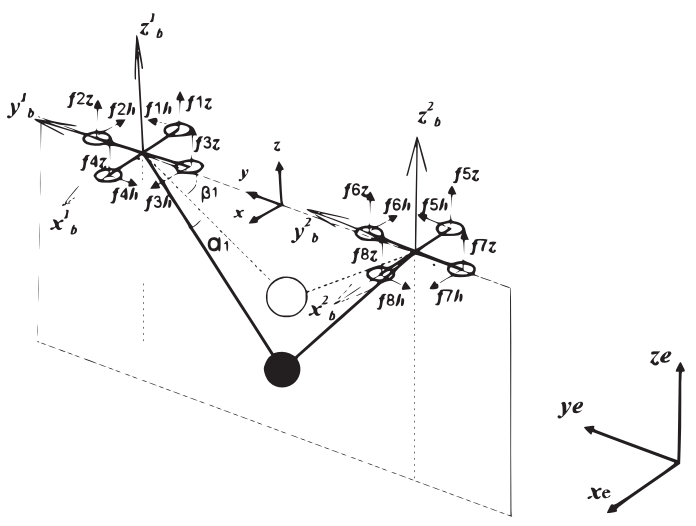

Fig. 1: Two quadrotors carrying a payload

In [10] an MPC controller was presented with PID for a quadrotor to track a predefined trajectory without constraints. An optimal flight control MPC for a quadrotor with a suspended load [11] was presented using visual feedback to compute the load position accurately. The state vector and control vector constraints were applied. In [12] an unconstrained MPC controller was proposed to track a trajectory for quadrotors based on three levels of control. By comparing the results of linear and nonlinear MPC control to track a trajectory using Micro Air Vehicles (MAVs) under wind disturbance [13], it shows that the difference between them is not significant.

Our previous works on single quadrotor in [14] and two quadrotors transporting a cable-suspended payload used an iterative LQR controller, which is an optimised solution for a non-linearity consideration, but without constraints. In this paper, we develop a constrained MPC controller for two quadrotors to transport a cable-suspended payload.

In the following, Section II presents the mathematical model of a cable suspended payload by two quadrotors. Section III illustrates the development of a constrained MPC controller. Section IV shows the simulation results. The conclusion and future work are given in Section V.

\section{Model Description}

The full system is presented in Fig. 1 including the inertial frame, intermediate frames, and body-fixed frames. The vertical and horizontal forces generated by each propeller and swing angles of the cable with respect to the intermediate frame are also shown where the intermediate frames are the translation results from the inertial frame to the centres of quadrotors. See table I for details. In order to simplify the problem, some reasonable assumptions are given as follows:

1) Each quadrotor is considered as a symmetrical rigid body.

2) The payload is considered as a point mass and is attached at the centre of each quadrotor.

3) The cable tension is always non-zero.

4) The air drag of the propellers is negligible.
TABLE I: Symbols and Definitions

\begin{tabular}{|c|c|}
\hline Symbol & Description \\
\hline$S_{e}: x_{e} y_{e} z_{e}$ & Inertial frame \\
\hline$S^{k}: x y z$ & $\begin{array}{l}\text { Intermediate frame: translation from } S_{e} \\
\text { to the center of the } i \text { th quadrotors } \\
k=1,2\end{array}$ \\
\hline$S_{b}^{k}: x_{b}^{k} y_{b}^{k} z_{b}^{k}$ & Body-fixed frame for quadrotor, \\
\hline $\boldsymbol{E}_{i} \in \mathbb{R}^{3}, i=1,2,3$ & Unit orthogonal vectors of $S_{e}$ \\
\hline$e_{i}^{k} \in \mathbb{R}^{3}, i=1,2,3$ & Unit orthogonal vectors of $S_{b}^{k}$ \\
\hline$\eta^{k}=[\phi, \theta, \psi]^{T} \in \mathbb{R}^{3}$ & $\begin{array}{l}\text { Euler angles of quadrotor defined } \\
\text { in } Z-Y-X\end{array}$ \\
\hline $\boldsymbol{T}_{e 2 b}^{k} \in \mathbb{R}^{3 \times 3}$ & Transformation matrix from $S_{e}$ to $S_{b}^{k}$ \\
\hline$\Omega^{k} \in \mathbb{R}^{3}$ & Angular velocity of quadrotor in $S_{b}^{k}$ \\
\hline$m_{Q}^{k}$ & Mass of the quadrotor \\
\hline$m_{P}$ & Mass of the payload \\
\hline$I_{Q}^{k} \in \mathbb{R}^{3}$ & $\begin{array}{l}\text { Inertial matrix of the quadrotor } \\
\text { with respect to } S_{b}^{k}\end{array}$ \\
\hline$\xi_{Q}^{k} \in \mathbb{R}^{3}$ & Position of the center of quadrotor in $\boldsymbol{S}_{\boldsymbol{e}}$ \\
\hline$\xi_{P} \in \mathbb{R}^{3}$ & Position of the payload in $S_{e}$ \\
\hline$x_{P}, y_{P}, z_{P}$ & Three elements of $\xi_{P}$ \\
\hline$L_{r}^{k}$ & Length of the rope \\
\hline$L_{Q}^{k}$ & Length of the quadrotor arm \\
\hline$\alpha^{k}, \beta^{k} \in \mathbb{R}$ & Angles of the rope with respect to $S^{k}$ \\
\hline$\rho^{k} \in \mathbb{R}^{3}$ & $\begin{array}{l}\text { Unit vector from the payload to } \\
\text { the attached point }\end{array}$ \\
\hline $\begin{array}{l}\boldsymbol{f}_{i z}^{k}, \boldsymbol{f}_{i h}^{k} \in \mathbb{R}^{3} \\
\operatorname{dir}\end{array}$ & $\begin{array}{l}\text { Vertial and horizontal forces generated } \\
\text { by } i \text { th propeller, } i=1,2,3,4\end{array}$ \\
\hline$k_{F}, k_{M} \in \mathbb{R}$ & Propeller aerodynamic parameters \\
\hline
\end{tabular}

Some symbols and acronyms used in this paper are listed in table I. The following relationships are available.

$$
\begin{aligned}
\boldsymbol{\rho}^{k} & =\left[-\sin \left(\beta^{k}\right),-\cos \left(\alpha^{k}\right) \cos \left(\beta^{k}\right), \sin \left(\alpha^{k}\right) \cos \left(\beta^{k}\right)\right]^{T} \\
\boldsymbol{\xi}_{P} & =x_{P} \boldsymbol{E}_{\mathbf{1}}+y_{P} \boldsymbol{E}_{\mathbf{2}}+z_{P} \boldsymbol{E}_{\mathbf{3}} \\
\boldsymbol{\xi}_{Q}^{k} & =\boldsymbol{\xi}_{P}+L_{r}^{k} \boldsymbol{\rho}^{k} \\
\boldsymbol{\Omega}^{k} & =\left[\begin{array}{ccc}
1 & 0 & -\sin \left(\theta^{k}\right) \\
0 & \cos \left(\phi^{k}\right) & \sin \left(\phi^{k}\right) \cos \left(\theta^{k}\right) \\
0 & -\sin \left(\phi^{k}\right) & \cos \left(\phi^{k}\right) \cos \left(\theta^{k}\right)
\end{array}\right]\left[\begin{array}{c}
\dot{\phi}^{k} \\
\dot{\theta^{k}} \\
\dot{\psi^{k}}
\end{array}\right]
\end{aligned}
$$

\section{A. Euler-Lagrange Equation}

The quadrotor-payload system has 13 degrees of freedom. Choosing $\boldsymbol{q}=$ $\left[x_{P}, y_{P}, z_{P}, \alpha^{1}, \beta^{1}, \phi^{1}, \theta^{1}, \psi^{1}, \alpha^{2}, \beta^{2}, \phi^{2}, \theta^{2}\right.$,

$\left.\psi^{2}\right]^{T}$ as the generalized coordinates will not only be convenient for controlling the trajectory of the payload but be helpful for extending to multi-vehicle situations. As a result, the Lagrangian $\mathbf{L}$ is composed by subtracting the kinetic energy $\mathbf{T}$ from the potential energy $\mathbf{U}$ and clarified in the equations below

$$
\begin{aligned}
& \mathbf{T}=\frac{1}{2} m_{P}\left(\dot{\boldsymbol{\xi}}_{P}\right)^{T} \cdot \dot{\boldsymbol{\xi}}_{P}+\frac{1}{2} m_{Q}^{1}\left(\dot{\boldsymbol{\xi}}_{Q}^{1}\right)^{T} \cdot \boldsymbol{\xi}_{Q}^{1}+\frac{1}{2}\left(\boldsymbol{\Omega}^{1}\right)^{T} \boldsymbol{I}_{Q}^{1} \boldsymbol{\Omega}^{1} \\
& \quad \frac{1}{2} m_{Q}^{2}\left(\dot{\boldsymbol{\xi}}_{Q}^{2}\right)^{T} \cdot \dot{\boldsymbol{\xi}}_{Q}^{2}+\frac{1}{2}\left(\boldsymbol{\Omega}^{2}\right)^{T} \boldsymbol{I}_{Q}^{2} \boldsymbol{\Omega}^{2} \\
& \mathbf{U}=m_{P} g \boldsymbol{\xi}_{P} \cdot \boldsymbol{E}_{3}+m_{Q}^{1} g \boldsymbol{\xi}_{Q}^{1} \cdot \boldsymbol{E}_{3}+m_{Q}^{2} g \boldsymbol{\xi}_{Q}^{2} \cdot \boldsymbol{E}_{3} \\
& \mathbf{L}=\mathbf{T}-\mathbf{U}
\end{aligned}
$$


Then the Euler-Lagrange equation is

$$
\frac{d}{d t}\left(\frac{\partial \mathbf{L}}{\partial \dot{\boldsymbol{q}}}\right)-\frac{\partial \mathbf{L}}{\partial \boldsymbol{q}}=\boldsymbol{Q}
$$

The generalized force $\boldsymbol{Q}$ defined here is based on the choice of the generalized coordinates $\boldsymbol{q}$ and the external conservative forces $\boldsymbol{F}_{i}$. For each quadrotor, the force $\boldsymbol{F}_{i}$ consists of two complements $\boldsymbol{f}_{i z}$ and $\boldsymbol{f}_{i h}$ (equation (4)) which are related with the angular speed $\omega_{i}$ of $i$ th propeller [14].

$$
\begin{aligned}
\boldsymbol{F}_{i}^{k} & =\boldsymbol{f}_{i z}^{k}+\boldsymbol{f}_{i h}^{k}, i=1,2,3,4 \\
\boldsymbol{f}_{i z}^{k} & =k_{F} \omega_{i}^{2} \boldsymbol{e}_{3}^{k}, i=1,2,3,4 \\
\boldsymbol{f}_{1 h}^{k} & =k_{M} \omega_{1}^{2} \boldsymbol{e}_{2}^{k} \\
\boldsymbol{f}_{2 h}^{k} & =k_{M} \omega_{2}^{2} \boldsymbol{e}_{1}^{k} \\
\boldsymbol{f}_{3 h}^{k} & =-k_{M} \omega_{3}^{2} \boldsymbol{e}_{2}^{k} \\
\boldsymbol{f}_{4 h}^{k} & =-k_{M} \omega_{4}^{2} \boldsymbol{e}_{1}^{k}
\end{aligned}
$$

where $\boldsymbol{e}_{i}^{k}=\boldsymbol{T}_{e 2 b}^{k} \boldsymbol{E}_{i}, i=1,2,3$.

As seen in Fig. 1, the point where the force $\boldsymbol{F}_{i}^{k}$ is applied on is the centre of each propeller and the corresponding position vector is noted as $\boldsymbol{\xi}_{i}^{k}$ seen in equation (5).

$$
\begin{aligned}
\boldsymbol{\xi}_{1}^{k} & =\boldsymbol{\xi}_{Q}^{k}+L_{Q} \boldsymbol{e}_{1}^{k} \\
\boldsymbol{\xi}_{2}^{k} & =\boldsymbol{\xi}_{Q}^{k}+L_{Q} \boldsymbol{e}_{2}^{k} \\
\boldsymbol{\xi}_{3}^{k} & =\boldsymbol{\xi}_{Q}^{k}-L_{Q} \boldsymbol{e}_{1}^{k} \\
\boldsymbol{\xi}_{4}^{k} & =\boldsymbol{\xi}_{Q}^{k}-L_{Q} \boldsymbol{e}_{2}^{k}
\end{aligned}
$$

According to the principle of virtual work, the generalized forces are given by equation (6) [14].

$$
Q_{i}=\sum_{j=1}^{4} \sum_{k=1}^{2} \frac{\partial\left(\boldsymbol{F}_{j}^{k} \cdot \boldsymbol{\xi}_{j}^{k}\right)}{\partial q_{i}}, i=1,2, . ., 13
$$

Taking the generalized forces and equation (2) into equation (3), the Euler-Lagrange equation can be rewritten in

$$
M \ddot{\boldsymbol{q}}=f(\boldsymbol{q}, \dot{\boldsymbol{q}})
$$

In the trajectory tracking control, the operating point is considered as the balance situation where the Euler angles of both quadrotors equal to zero and the cable angles equal to the designed constants. The state $\boldsymbol{x}$ and control $\boldsymbol{u}$ of the system are defined as below:

$$
\begin{aligned}
\boldsymbol{x}= & {\left[x_{P}, \dot{x}_{P}, y_{P}, \dot{y}_{P}, z_{P}, \dot{z}_{P}, \alpha^{1}, \dot{\alpha}^{1}, \beta^{1}, \dot{\beta}^{1}, \phi^{1}, \dot{\phi}^{1}, \theta^{1},\right.} \\
& \left.\dot{\theta}^{1}, \psi^{1}, \dot{\psi}^{1}, \alpha^{2}, \dot{\alpha}^{2}, \beta^{2}, \dot{\beta}^{2}, \phi^{2}, \dot{\phi}^{2}, \theta^{2}, \dot{\theta}^{2}, \psi^{2}, \dot{\psi}^{2}\right]^{T} \in \mathbb{R}^{26} \\
\boldsymbol{u}= & {\left[\omega_{1}, \omega_{2}, \omega_{3}, \omega_{4}, \omega_{5}, \omega_{6}, \omega_{7}, \omega_{8}\right]^{T} \in \mathbb{R}^{8} }
\end{aligned}
$$

The linearised model is obtained in equation (8).

$$
\ddot{\boldsymbol{q}}=\left.\boldsymbol{M}^{-1} \frac{\partial f}{\partial \boldsymbol{x}}\right|_{\boldsymbol{x}_{0}} \Delta \boldsymbol{x}+\left.\boldsymbol{M}^{-1} \frac{\partial f}{\partial \boldsymbol{u}}\right|_{\boldsymbol{u}_{0}} \Delta \boldsymbol{u}
$$

Furthermore, equation (8) can be transformed into a discrete form.

$$
x_{k+1}=A_{k} x_{k}+B_{k} u_{k}
$$

where $\boldsymbol{A}_{\boldsymbol{k}} \in \mathbb{R}^{26 \times 26}, \boldsymbol{B}_{\boldsymbol{k}} \in \mathbb{R}^{26 \times 8}$.

\section{Constrained Linear Model Predictive Control}

Model predictive control (MPC) is a worth describing method based on the principle of prediction to a finite horizon at each sample starting from the current state. Subjected to the state and control constraints, MPC is solved to determine the optimal control input sequence over the finite horizon. Then, it implements the first control input in the optimised control input sequence. The discrete-time dynamical model description for the system with two quadrotors carrying a cable-suspended payload is shown in equation (9).

The finite horizon optimal control solves the following constrained optimisation problem at each time instant $k$ to implement the MPC algorithm. The cost function is presented by:

$$
\begin{aligned}
J= & \left(x_{N}-x_{N}^{*}\right)^{T} Q_{f}\left(x_{N}-x_{N}^{*}\right) \\
& +\sum_{t=k}^{N+k-1}\left[\left(x_{t}-x_{t}^{*}\right)^{T} Q\left(x_{t}-x_{t}^{*}\right)+u_{t}^{T} R u_{t}\right]
\end{aligned}
$$

where the terminal state and its desired state are denoted by $\boldsymbol{x}_{\boldsymbol{N}}$ and $\boldsymbol{x}_{\boldsymbol{N}}^{*}$, respectively, and the reference state is denoted by $\boldsymbol{x}_{\boldsymbol{k}}^{*}$. The prediction horizon is denoted by $\boldsymbol{N}$. $\boldsymbol{Q}_{\boldsymbol{f}}$ and $\boldsymbol{Q}$ are presented by positive semidefinite state matrices and $\boldsymbol{R}$ is presented by positive definite matrix.

The constrained optimisation problem (COP) at each time instant $k$ is

$$
\min _{\boldsymbol{u}_{k}} J
$$

Subject to

$$
\begin{aligned}
& \boldsymbol{x}_{\boldsymbol{k}+\mathbf{1}}=\boldsymbol{A}_{\boldsymbol{k}} \boldsymbol{x}_{\boldsymbol{k}}+\boldsymbol{B}_{\boldsymbol{k}} \boldsymbol{u}_{\boldsymbol{k}} \\
& \boldsymbol{x}_{\boldsymbol{k}} \in \mathbb{X}, \text { the state constraints } \\
& \boldsymbol{u}_{\boldsymbol{k}} \in \mathbb{U} \text {, the control constraints }
\end{aligned}
$$

where the state and input constraints are presented as

$$
\begin{aligned}
& \mathbb{X}=\left\{\boldsymbol{x}_{\text {kmin }} \leq \boldsymbol{x}_{\boldsymbol{k}} \leq \boldsymbol{x}_{\text {kmax }}\right\} \\
& \mathbb{U}=\left\{\boldsymbol{u}_{\text {kmin }} \leq \boldsymbol{u}_{\boldsymbol{k}} \leq \boldsymbol{u}_{\text {kmax }}\right\}
\end{aligned}
$$

A quadratic programming (QP) algorithm can be used to solve the COP problem at each time instant $\boldsymbol{k}$, which can produce an input sequence $\left\{\boldsymbol{u}_{\boldsymbol{k} \mid \boldsymbol{k}}, \boldsymbol{u}_{\boldsymbol{k}+\mathbf{1} \mid \boldsymbol{k}} \cdots, \boldsymbol{u}_{\boldsymbol{k}+\boldsymbol{N}-1 \mid \boldsymbol{k}}\right\}$. Then the true input at $\boldsymbol{k}$ is $\boldsymbol{u}_{\boldsymbol{k}}=\boldsymbol{u}_{\boldsymbol{k} \mid \boldsymbol{k}}$. 
TABLE II: The used parameters for the system model

\begin{tabular}{lclc}
\hline Symbol & Definition & Value & Units \\
\hline $\boldsymbol{I}_{Q x}^{k}$ & Roll Inertial & $4.4 \times 10^{-} 3$ & $\mathrm{~kg} \cdot \mathrm{m}^{2}$ \\
\hline $\boldsymbol{I}_{Q y}^{k}$ & Pitch Inertial & $4.4 \times 10^{-} 3$ & $\mathrm{~kg} \cdot \mathrm{m}^{2}$ \\
\hline $\boldsymbol{I}_{Q z}^{k}$ & Yaw Inertial & $8.8 \times 10^{-} 3$ & $\mathrm{~kg} \cdot \mathrm{m}^{2}$ \\
\hline$m_{Q}^{1}$ & Mass & 0.55 & $\mathrm{~kg}$ \\
\hline$m_{Q}^{2}$ & Mass & 0.55 & $\mathrm{~kg}$ \\
\hline$m_{P}$ & Mass & 0.2 & $\mathrm{~kg}$ \\
\hline$g$ & Gravity & 9.81 & $\mathrm{~m} / \mathrm{s}^{2}$ \\
\hline$L_{Q}^{k}$ & Arm Length & 0.17 & $\mathrm{~m}$ \\
\hline$L_{r}^{k}$ & Rope Length & 1 & $\mathrm{~m}$ \\
\hline$I_{r}$ & Rotor Inertial & $4.4 \times 10^{-} 5$ & $\mathrm{~kg} \cdot \mathrm{m}^{2}$ \\
\hline
\end{tabular}

\section{Simulation Results}

The simulation is conducted in MATLAB. The applied parameters for this simulation are shown in table II. We compare the control performance of the MPC controller and an LQR controller to demonstrate how the constraints on state and control vectors affect the performance.

The tracking control simulation results of the suspended payload with two quadrotors by cables are verified by utilizing the desired trajectory, which is a 3D-spiral trajectory. The simulation on an eight shape trajectory is also conducted.

The desired load 3D spiral trajectory is defined by $x^{*}=\cos (2 \pi / t), y^{*}=\sin (2 \pi / t), z^{*}=0.1 * t$, where $t$ is started from 0 with a sampling time of $0.2 \mathrm{~s}$, and the tracking simulation is up to $30 \mathrm{~s}$. The MPC prediction horizon is selected as 10 . The operating point of two quadrotors with suspended load is made with rope angles $\alpha_{1}=90^{\circ}, \beta_{1}=45^{\circ}, \alpha_{2}=90^{\circ}, \beta_{2}=-45^{\circ}$. The weight matrices of the cost function are selected as

$$
\begin{gathered}
Q=Q_{N}=\operatorname{diag}([1000,1,1000,1,1000,1,1,1,1,1,1,1,1,1, \\
1,1,1,1,1,1,1,1,1,1,1,1]) \\
R=\operatorname{diag}([0.0001,0.001,0.0001,0.001,0.0001,0.001, \\
0.0001,0.001])
\end{gathered}
$$

With regard to the constraints on control vectors, the maximum saturation limit is considered to reflect the limitation of practical rotor's power. They are presented by the maximum angular velocity for all the rotors in both quadrotors $\boldsymbol{F}_{i}^{k} \leq 700 \mathrm{rad} / \mathrm{s}$.

With regard to the constraints on state vectors, the maximum and minimum limits are considered to reflect the limitation of the environment. This includes the payload position limit in $x$ direction, and the payload swing angle limit to avoid the possible collisions. The desired load trajectory is shown in Fig. 2 represented by the red trajectory starting from $(1,0,0)$. The constraints includes $x_{k} \leq-0.5$ and $88^{\circ} \leq \alpha 1, \alpha 2 \leq 92^{\circ}, 40^{\circ} \leq \beta 1, \beta 2 \leq 50^{\circ}$.

The MPC trajectory is shown in Fig. 2 represented by the blue trajectory. It starts from $(0,0,0)$, then moves

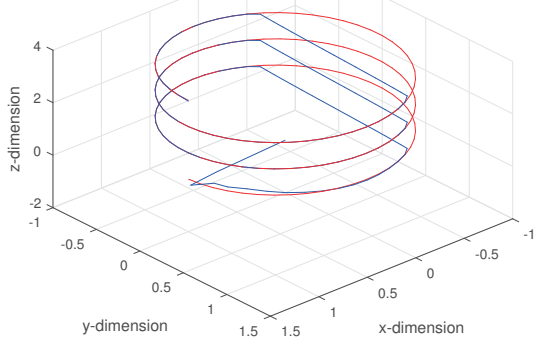

Fig. 2: 3D load position using the constrained MPC controller

towards the desired initial point $(1,0,0)$ and closely tracks the desired.

The comparisons between the MPC and LQR controller on the payload position and the Eular angles of the first quadrotor are shown in Figs. 7, 8, 9, and 10. Figs. 7 and 8 show the MPC controller performs better on tracking the desired ones, and constraining the position than the LQR controller. Figs. 9 and 10 show the MPC controller has a smoother pose than the LQR controller.

The desired load eight-shape trajectory is shown in red in Figs 11 and 12. The desired initial position is $(0,0,0)$. The LQR tracking performance is shown in Fig. 11. It can be seen that the LQR tracking trajectory tries to move closer to the desired one, but fails due to the constraint imposed on the thrust. Further, we can not impose the constraints on the state variables. The MPC tracking trajectory is shown in Fig. 12 where the input thrust and state constraints, including $x_{k} \leq-0.5$ and $88^{\circ} \leq$ $\alpha 1, \alpha 2 \leq 92^{\circ}, 40^{\circ} \leq \beta 1, \beta 2 \leq 50^{\circ}$ are imposed. It has a very slight overshoot from the desired one short time at the initial position. Then the tracking performance works very well. The $x$ direction constraint is clearly observed and respected. In general, a much better performance has been demonstrated by the MPC controller when compared with the LQR controller.

The LQR trajectory is shown in Fig. 3 represented by the blue trajectory. It starts from $(0,0,0)$, then moves towards the desired one, but fails to move close to it due to the limits applied to their angular velocities.

Four angular velocities of the first quadrotor using the MPC controller is shown in Fig. 4. It is clearly indicated that all of them are capped at $700 \mathrm{rad} / \mathrm{s}$, i.e. the constraints on control vector are respected. Four angular velocities of the second quadrotor have a similar performance and are ignored here.

The rope angles with the first quadrotor using the MPC controller and the LQR controller are shown in Figs. 5 and 6 , respectively. It can be seen that the constraints on the angles are respected by the MPC controller, but not by the LQR controller. 


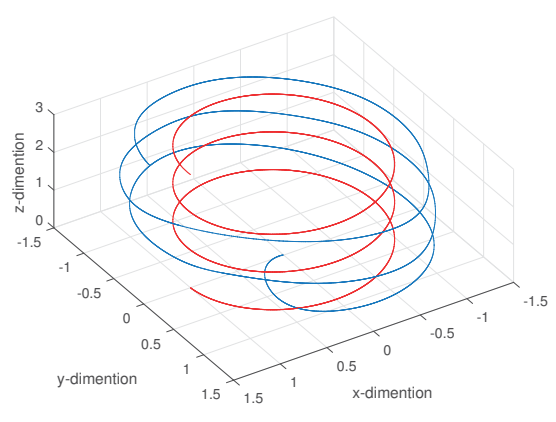

Fig. 3: 3D load position using the LQR controller with the control limitation

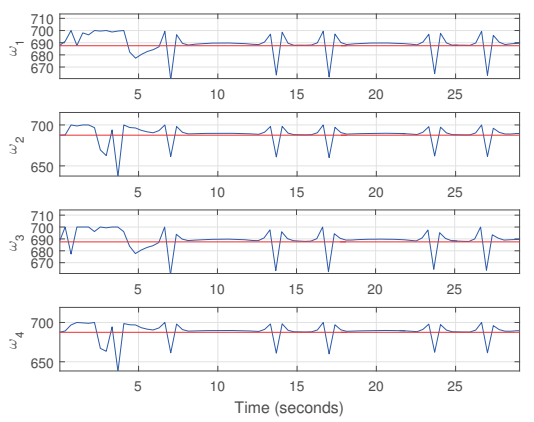

Fig. 4: The angular velocities of the first quadrotor using MPC controller

\section{Conclusion}

In this paper, a constrained MPC controller is applied for tracking control of a cable suspended payload with two quadrotors. A nonlinear model of the system is developed and linearized. The constraints on state and control vectors are considered. We evaluate our model and the MPC controller using simulation and compare the performance of the constrained MPC controller and the LQR controller. The simulation results show the MPC controller is better in controlling the system than the
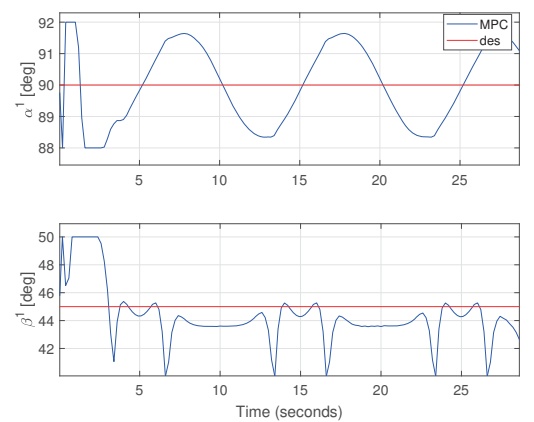

Fig. 5: The rope angles with the first quadrotor using the MPC controller
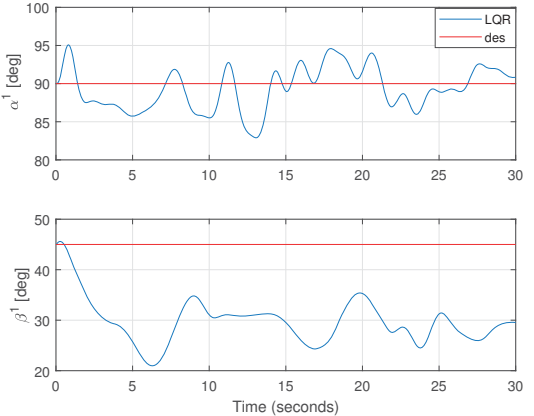

Fig. 6: The rope angles with the first quadrotor using the LQR controller
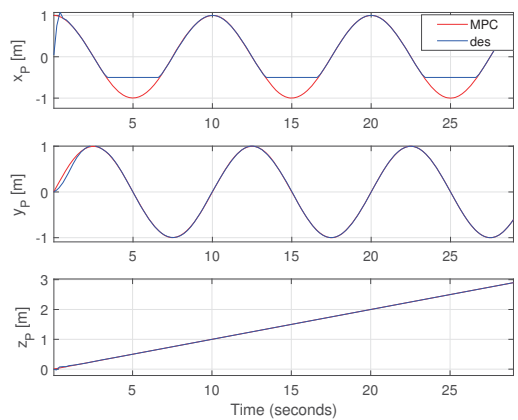

Fig. 7: The payload position using the MPC controller
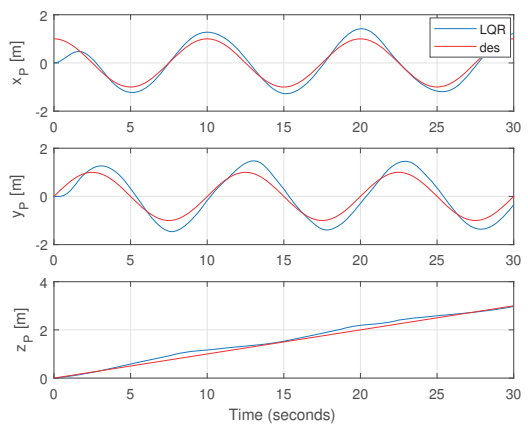

Fig. 8: The payload position using the LQR controller
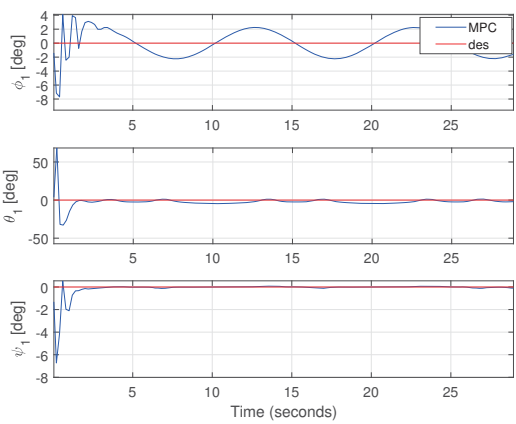

Fig. 9: The Eular angles of the first quadrotor using the MPC controller 

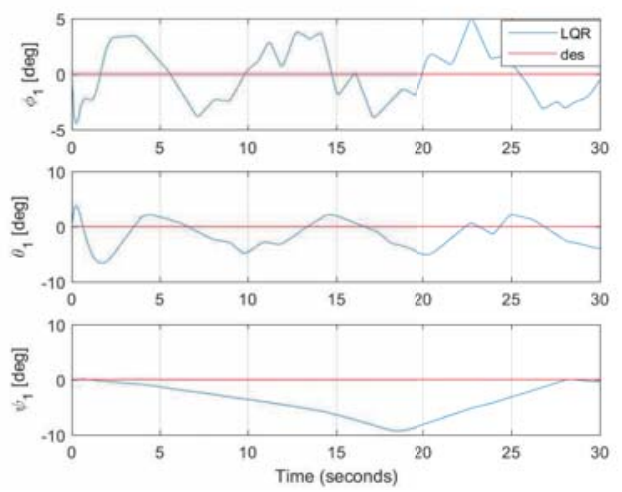

Fig. 10: The Eular angles of the first quadrotor using the LQR controller

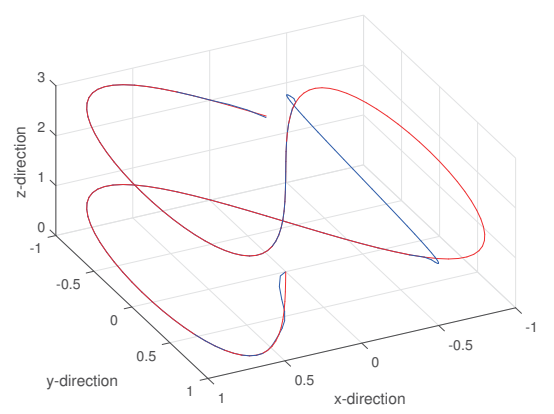

Fig. 11: 3D load position using the constrained MPC controller

LQR controller under the consideration of constraints. The constraints are an important factor in applying a controller to practical systems. Our next step work will be focused on the implementation of the constrained MPC controller on a practical system.

\section{REFERENCES}

[1] G. Wu and K. Sreenath, "Geometric control of multiple quadrotors transporting a rigid-body load," in 53rd IEEE Conference on Decision and Control. IEEE, 2014, pp. 6141-6148.
[2] T. Lee, K. Sreenath, and V. Kumar, "Geometric control of cooperating multiple quadrotor uavs with a suspended payload," in 52nd IEEE Conference on Decision and Control. IEEE, 2013, pp. $5510-5515$.

[3] T. Lee, "Geometric control of multiple quadrotor uavs transporting a cable-suspended rigid body," in 53rd IEEE Conference on Decision and Control. IEEE, 2014, pp. 6155-6160.

[4] K. Alexis, G. Nikolakopoulos, and A. Tzes, "Experimental model predictive attitude tracking control of a quadrotor helicopter subject to wind-gusts," in Control $\&$ Automation (MED), 2010 18th Mediterranean Conference on. IEEE, 2010, pp. 14611466.

[5] B. Yu, Y. Zhang, I. Minchala, and Y. Qu, "Fault-tolerant control with linear quadratic and model predictive control techniques against actuator faults in a quadrotor uav," in Control and Fault-Tolerant Systems (SysTol), 2013 Conference on. IEEE, 2013, pp. 661-666.

[6] M. Iskandarani, S. N. Givigi, C. A. Rabbath, and A. Beaulieu, "Linear model predictive control for the encirclement of a target using a quadrotor aircraft," in Control \& Automation (MED), 2013 21st Mediterranean Conference on. IEEE, 2013, pp. 15501556.

[7] K. Alexis, G. Nikolakopoulos, and A. Tzes, "Model predictive control scheme for the autonomous flight of an unmanned quadrotor," in Industrial Electronics (ISIE), 2011 IEEE International Symposium on. IEEE, 2011, pp. 2243-2248.

[8] R. Hedjar, "Robust one-step-ahead model predictive control of vtol-uavs," in Control and Decision Conference (CCDC), 2015 27th Chinese. IEEE, 2015, pp. 3053-3058.

[9] M. Iskandarani, S. N. Givigi, G. Fusina, and A. Beaulieu, "Unmanned aerial vehicle formation flying using linear model predictive control," in Systems Conference (SysCon), 2014 8th Annual IEEE. IEEE, 2014, pp. 18-23.

[10] A. S. Imam and R. Bicker, "Quadrotor model predictive flight control system," International Journal of Current Engineering and Technology, vol. 4, no. 1, pp. 355-365, 2014.

[11] M. Zürn, K. Morton, A. Heckmann, A. McFadyen, S. Notter, and F. Gonzalez, "Mpc controlled multirotor with suspended slung load: System architecture and visual load detection," in Aerospace Conference, 2016 IEEE. IEEE, 2016, pp. 1-11.

[12] M. Bangura and R. Mahony, "Real-time model predictive control for quadrotors," IFAC Proceedings Volumes, vol. 47, no. 3 , pp. $11773-11780,2014$.

[13] M. Kamel, M. Burri, and R. Siegwart, "Linear vs nonlinear mpc for trajectory tracking applied to rotary wing micro aerial vehicles," arXiv preprint arXiv:1611.09240, 2016.

[14] Y. Alothman and D. Gu, "Quadrotor transporting cablesuspended load using iterative linear quadratic regulator (ilqr) optimal control," in Computer Science and Electronic Engineering (CEEC), 2016 8th. IEEE, 2016, pp. 168-173.

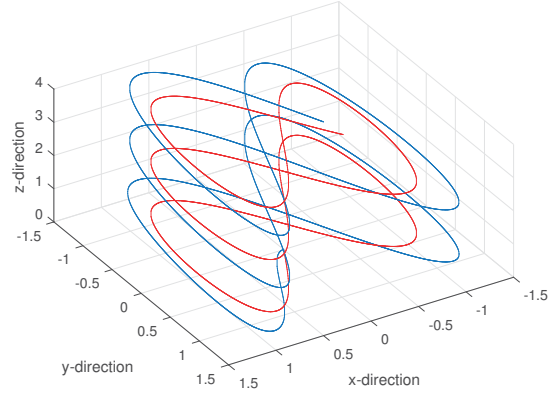

Fig. 12: 3D load position using the constrained LQR controller 